\title{
Breast-feeding and adherence to infant feeding guidelines do not influence bone mass at age 4 years
}

\author{
Nicholas C. Harvey, Sian M. Robinson, Sarah R. Crozier, Lynne D. Marriott, Catharine R. Gale, \\ Zoe A. Cole, Hazel M. Inskip, Keith M. Godfrey, Cyrus Cooper* and the Southampton Women's Survey \\ Study Group $\dagger$ \\ MRC Epidemiology Resource Centre, University of Southampton, Southampton General Hospital, Southampton SO16 6YD, UK \\ (Received 4 September 2008 - Revised 5 February 2009 - Accepted 4 March 2009 - First published online 2 April 2009)
}

The impact of variations in current infant feeding practice on bone mineral accrual is not known. We examined the associations between duration of breast-feeding and compliance with infant dietary guidelines and later bone size and density at age 4 years. At total of 599 (318 boys) mother-child pairs were recruited from the Southampton Women's Survey. Duration of breast-feeding was recorded and infant diet was assessed at 6 and 12 months using FFQ. At 6 and 12 months the most important dietary pattern, defined by principal component analysis, was characterised by high consumption of vegetables, fruits and home-prepared foods. As this was consistent with infant feeding recommendations, it was denoted the 'infant guidelines' pattern. At age 4 years, children underwent assessment of whole-body bone size and density using a Hologic Discovery dual-energy X-ray absorptiometry instrument. Correlation methods were used to explore the relationships between infant dietary variables and bone mineral. There was no association between duration of breast-feeding in the first year of life and 4-year bone size or density. 'Infant guidelines' pattern scores at 6 and 12 months were also unrelated to bone mass at age 4 years. We observed wide variations in current infant feeding practice, but these variations were not associated with differences in childhood bone mass at age 4 years.

Osteoporosis epidemiology: Growth: Infant diet: Guidelines: Peak bone mass

Little is known about the impact of early nutrition on later childhood bone health. Previous studies have focused on variations in milk-feeding, as formula milk and breast milk differ in composition, most notably in protein and vitamin D content. However, the findings of these studies are not consistent and bone mineral in breast-fed children has been shown to be both lower and higher than in those who were formula-fed. A study of forty infants found reduced whole-body bone mineral content (BMC) in the breast-fed $v$. formula-fed babies at 12 months $^{(1)}$. In contrast, amongst 330 eight-year-old children, those who had been breast-fed in infancy had higher bone mineral density (BMD) compared with those who had been formula-fed ${ }^{(2)}$. Previous work has also revealed uncertainty regarding the long-term impact of breast- $v$. formula-feeding, although the few studies with greater duration of follow-up suggest that any short-term differences are ameliorated by later childhood $^{(3,4)}$.

There are large variations in infant feeding practice in the $\mathrm{UK}^{(5,6)}$ but little is known about the role of the weaning diet in relation to later bone health. These variations need to be considered. First, there may be confounding effects on the associations between bone health and variations in milk-feeding, as the duration of breast-feeding is related both to the age when solid foods are introduced ${ }^{(6)}$ and the types of solid foods fed during weaning ${ }^{(5-7)}$. Second, in a recent study we have shown that variations in bone health in children are associated with the dietary patterns of their mothers in pregnancy ${ }^{(8)}$. In a cohort of children aged 9 years, those who were born to mothers who had a healthy 'prudent' pattern of diet in pregnancy (characterised by greater consumption of fruit, vegetables and wholemeal bread) had a greater bone size and $\mathrm{BMD}^{(8)}$. These relationships were independent of a range of confounding factors including vitamin D status of the mother in late pregnancy. Since the dietary patterns of mothers and infants are highly correlated $^{(5)}$ it is possible that variations in the weaning diet are also related to later bone status.

In the present study, we assessed the relationship between childhood bone size and density at age 4 years with the duration and type of milk-feeding in infancy, and compliance with infant feeding guidance. We also examined whether any associations between infant feeding and bone status were independent of the effects of maternal diet.

Abbreviations: BMC, bone mineral content; BMD, bone mineral density; DXA, dual-energy X-ray absorptiometry; SWS, Southampton Women's Survey.

* Corresponding author: Professor Cyrus Cooper, fax +44 238070 4021, email cc@mrc.soton.ac.uk

$\dagger$ Southampton Women's Survey Study Group: David J. P. Barker, Catherine M. Law, Vanessa A. Cox, Elaine M. Dennison, Pat Taylor, Patricia J. Coakley and Julia Hammond. 


\section{Methods}

The Southampton Women's Survey

The Southampton Women's Survey (SWS) is a study of a population sample of non-pregnant women aged 20-34 years, resident in the city of Southampton (Hants, UK) ${ }^{(9)}$. The SWS started in 1998. Its aim is to identify the maternal influences acting before and during pregnancy that determine fetal growth, and to characterise how maternal and intra-uterine influences interact with the offspring's genes and postnatal environment to determine subsequent growth, development and health. Assessments of lifestyle, diet ${ }^{(10)}$ and anthropometry were performed at study entry and then in early (11 weeks) and late (34 weeks) gestation in those women who became pregnant.

\section{Dietary assessment}

Children born to women in the SWS were followed-up at 6 months of age (within 2 weeks of their 6-month birthday) and again at 12 months (within a period 2 weeks before and 3 weeks after their 12-month birthday). Diet was assessed at 6 months and at 12 months using administered FFQ that were developed for the $\operatorname{SWS}^{(11,12)}$. Trained research nurses administered the FFQ. The average frequency of consumption and amounts consumed of the listed foods over the $7 \mathrm{~d}$ (at 6 months) or month (at 12 months) preceding the visit were recorded. Using principal component analysis ${ }^{(13)}$, the most important pattern of diet we identified at both 6 and 12 months of age was characterised by a high frequency of consumption of vegetables, fruit, meat/fish and other homeprepared foods. It describes a pattern of foods that conforms to UK infant feeding guidelines ${ }^{(14)}$, and was called the 'infant guidelines' pattern. 'Infant guidelines' scores, that indicate the degree of compliance with this pattern, were calculated for all infants at 6 and 12 months of age. At both of these time-points, details of the milk-feeding history for the preceding 6 months were recorded; the duration of breast-feeding was defined according to the date of the last breast-feed. We define 'weaning' as the period of transition in infancy between a diet based on milk-feeding to one based on solid foods.

Maternal diet was assessed by FFQ before and during pregnancy $^{(10)}$. A principal component analysis of the mothers' pre-pregnant dietary data yielded a component that was characterised by high intakes of fruit, vegetables, wholemeal bread, rice and pasta, but low intakes of white bread, added sugar, and tinned vegetables, and describes a pattern of foods that reflects recommendations for a 'healthy' diet, called a 'prudent' diet ${ }^{(15)}$. Prudent diet scores were available for each mother before pregnancy; higher prudent diet scores indicate a 'healthier' pattern of eating.

\section{Four-year follow-up}

Subjects were recruited from the SWS cohort. The mother and child were invited to visit the Osteoporosis Centre at Southampton General Hospital for assessment of bone mass. At this visit written informed consent for the dual-energy X-ray absorptiometry (DXA) scan was obtained from the mother or father. The child's height (using a Leicester Height Measurer; Seca Ltd, Birmingham, UK) and weight (in underpants only, using calibrated digital scales; Seca Ltd, Birmingham, UK) were measured. A whole-body DXA scan was obtained, using a Hologic Discovery instrument (Hologic Inc., Bedford, MA, USA). To encourage compliance, a sheet with appropriate coloured cartoons was laid on the couch first; to help reduce movement artifact, the children were shown a suitable DVD cartoon. The total radiation dose for the scans was 4.7 microsieverts for whole-body measurement (paediatric scan mode). The manufacturer's $\mathrm{CV}$ for the instrument was $0.75 \%$ for whole-body BMD and the experimental CV when a spine phantom was repeatedly scanned in the same position sixteen times was $0.68 \%$. The instrument was calibrated using a spine phantom daily and a step phantom weekly. The ability of DXA to measure bone mass in small subjects was demonstrated by Abrams et al. ${ }^{(16)}$ using miniature piglets, where correlation between DXA-derived BMC and ashed Ca content was $0.90(P<0.001)$.

\section{Statistical analysis}

All variables were checked for normality. To test the difference in normally and non-normally distributed variables, $t$ tests and Mann-Whitney $U$ tests were used, respectively, by sex. Correlation and linear regression methods were used to explore the relationships between infant diet and childhood whole-body bone area, BMC and areal BMD using Stata V10.0 (Statacorp LP, College Station, TX, USA). Bone mineral measures included the whole body but excluded the head. To correct for body size, we used BMC adjusted for bone area, height and weight in a regression model, to give estimated volumetric BMD.

The present study was conducted according to the guidelines laid down in the Declaration of Helsinki and all procedures involving human subjects were approved by the Southampton and South West Hampshire Research Ethics Committee. Written informed consent was obtained from all subjects.

\section{Results}

\section{Characterisation of mothers and babies}

The characteristics of the mothers and children are shown in Tables 1 and 2. The mean age of the mothers at delivery of the baby was 30.4 (SD 3.8) years and $47 \%$ were in their first pregnancy; $11 \%$ continued to smoke in late pregnancy and $46 \%$ were in social classes I and II, $42 \%$ in class III and $11 \%$ in classes IV and V. Table 3 gives the distribution of duration of breast-feeding: $88 \%$ of women initiated breast-feeding and $29 \%$ were still breast-feeding when the

Table 1. Maternal characteristics ( $n$ 599)

(Mean values and standard deviations)

\begin{tabular}{|c|c|c|c|}
\hline & Mean & SD & $n$ \\
\hline Age (years) & 30.4 & 3.8 & 599 \\
\hline Height $(\mathrm{cm})$ & 163.7 & 6.6 & 595 \\
\hline BMI $\left(\mathrm{kg} / \mathrm{m}^{2}\right)$ & & & 594 \\
\hline Median & & \\
\hline Interquartile range & \multicolumn{2}{|c|}{$22 \cdot 2-27 \cdot 4$} & \\
\hline
\end{tabular}


Table 2. Anthropometric and skeletal characteristics of children aged 4 years $(n 599)^{\star}$ (Mean values and standard deviations)

\begin{tabular}{|c|c|c|c|c|c|}
\hline & \multicolumn{2}{|c|}{ Boys (n 318) } & \multicolumn{2}{|c|}{ Girls (n 281) } & \multirow[b]{2}{*}{$P$ difference } \\
\hline & Mean & SD & Mean & SD & \\
\hline Age (years) & & & & & 0.804 \\
\hline Median & \multirow{2}{*}{\multicolumn{2}{|c|}{$\begin{array}{c}4 \cdot 1 \\
4 \cdot 1-4 \cdot 2\end{array}$}} & \multirow{2}{*}{\multicolumn{2}{|c|}{$\begin{array}{c}4 \cdot 1 \\
4 \cdot 1-4 \cdot 2\end{array}$}} & \\
\hline Interquartile range & & & & & \\
\hline Birth weight $(\mathrm{g}) \dagger$ & 3596 & 472 & 3481 & 436 & 0.002 \\
\hline Bone area $\left(\mathrm{cm}^{2}\right)$ & 749 & 44 & 767 & 52 & $<0.0001$ \\
\hline $\mathrm{BMC}(\mathrm{g})$ & 371 & 43 & 378 & 49 & 0.084 \\
\hline Areal BMD $\left(\mathrm{g} / \mathrm{cm}^{2}\right)$ & 0.494 & 0.03 & 0.491 & 0.04 & 0.234 \\
\hline Volumetric BMD $\left(\sim \mathrm{g} / \mathrm{cm}^{3}\right)$ & 375 & 16 & 372 & 19 & 0.029 \\
\hline Infant guidelines at 6 months & 0.026 & 0.995 & 0.044 & 0.952 & 0.824 \\
\hline Infant guidelines at 12 months & 0.086 & 0.974 & 0.146 & 0.958 & 0.461 \\
\hline
\end{tabular}

BMC, bone mineral content; BMD, bone mineral density.

${ }^{*}$ All measures of bone mass are for whole body minus head.

† Birth weight adjusted for gestational age.

infant was more than 6 months old. The mothers were broadly similar to mothers whose children did not undergo DXA assessment, but were less likely to smoke, but more likely to have a higher educational status and to breast-feed for longer $(P<0 \cdot 01)$. After exclusion of twenty-two children whose whole-body DXA scans showed excessive movement artifact, there were 318 boys and 281 girls at age 4 years. The boys were of higher birth weight and bone area $(P<0.01)$ than the girls; values were adjusted for sex in subsequent analyses. The dietary pattern scores did not differ significantly between male and female children.

\section{Infant feeding bone size and density at age 4 years}

Duration of breast-feeding did not predict any measure of bone size or density in the children at age 4 years and there was thus no difference in bone mineral at age 4 years between predominantly breast- and formula-fed infants. Fig. 1(a)-(d) shows the relationship between duration of breast-feeding and offspring whole-body bone area (Fig. 1(a)), BMC (Fig. 1(b)), areal BMD (Fig. 1(c)) or volumetric BMD (Fig. 1(d)) at 4 years. Comparing those who received breast milk for less than 1 month or not at all with those who were breast-fed for 2-6 months, there were no differences in childhood bone size or density $(P>0 \cdot 05)$.

We found no statistically significant associations between infant guidelines score at age 6 or 12 months and childhood bone size or density at age 4 years; the 12-month data are summarised in Fig. 1(e)-(h). Inclusion of childhood milk intake and

Table 3. Distribution of duration of breast-feeding among infants born to 597 mothers

\begin{tabular}{lrr}
\hline Duration of breast-feeding (completed months) & $n$ & $\%$ \\
\hline Never tried & 72 & 12 \\
$<1$ month & 122 & 20 \\
$1-3$ months & 106 & 18 \\
$4-6$ months & 102 & 21 \\
$7-11$ months & 96 & 16 \\
12 or more months & 78 & 13 \\
Total & 597 & 100 \\
\hline
\end{tabular}

maternal birth weight, social class, mother's prudent diet score, parity, physical activity (measured by walking speed), body build (triceps skinfold thickness) and smoking in bivariate and then multivariate models did not alter these findings.

\section{Discussion}

We have shown, in a large, population-based, prospective study, that there is no association between duration of breast-feeding or adherence to current guidelines on infant feeding, and bone mineral accrual in early childhood. These findings were not modified by inclusion of maternal lifestyle and anthropometric factors or childhood milk intake.

The present study had a prospective design, with detailed characterisation of maternal and infant diet, and a validated measure of bone mass at 4 years. Because the children were visited by trained research nurses at 6 and 12 months of age, when contemporary information on milk-feeding and diet was obtained, this should minimise any effect of recall bias that may influence retrospective reports of infant feeding if determined at later ages. However, there were several limitations. First, diet was assessed using administered FFQ. Whilst there is concern that such questionnaires can be prone to measurement error, they have been shown to identify similar patterns of diet to dietary records ${ }^{(17,18)}$, and pattern scores determined using different dietary methods are highly correlated $^{(5,19)}$. In separate validation studies we have also established that nutrient intakes assessed using these FFQ at 6 and 12 months of age are comparable with nutrient intakes assessed using weighed records ${ }^{(11,12)}$. Second, the mothers of participants were a subset of the overall SWS cohort. These women were broadly similar to the other mothers, although they tended to smoke less and have a higher educational status. This could have reduced our power to demonstrate associations, as the spread of diet scores is likely to have been narrowed, but in fact the spread was similar in both groups (data not shown). Third, measurement of bone mineral in children is hampered by their tendency to move and also by their low absolute BMC. However, we used specific paediatric software, movement artifact was minimal and the small number of children with excess movement artifact were 
(a)

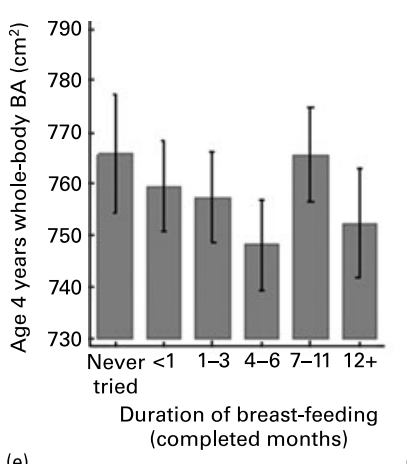

(e)

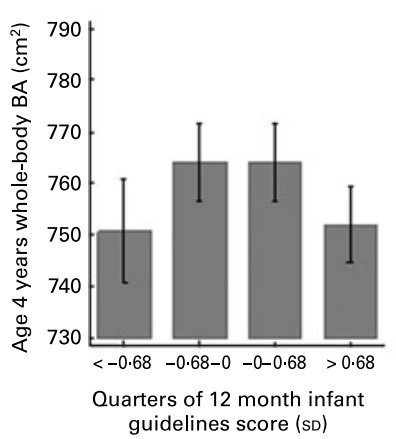

(b)

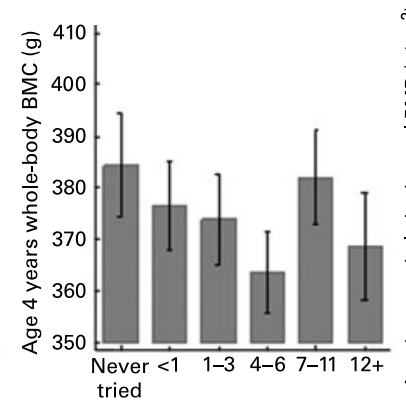

tried

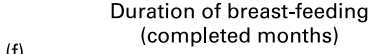

(f)

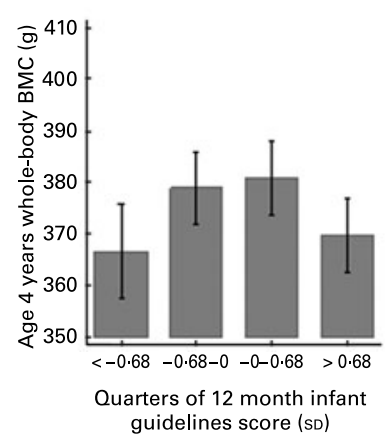

(c)

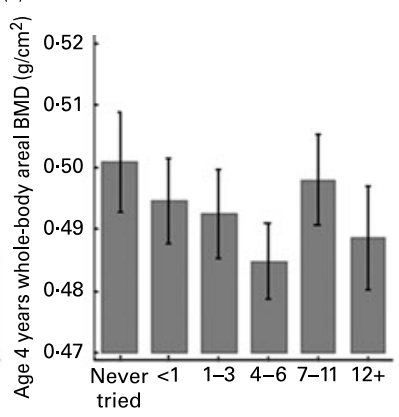

Duration of breast-feeding (completed months)

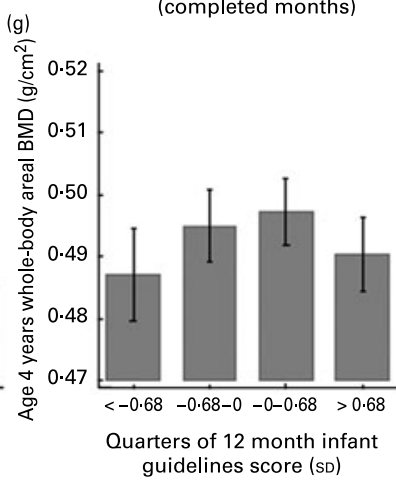

(d)

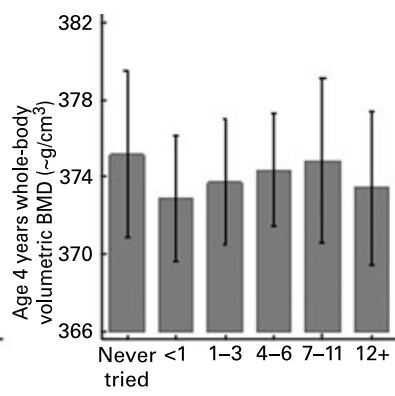

Duration of breast-feeding

(h)

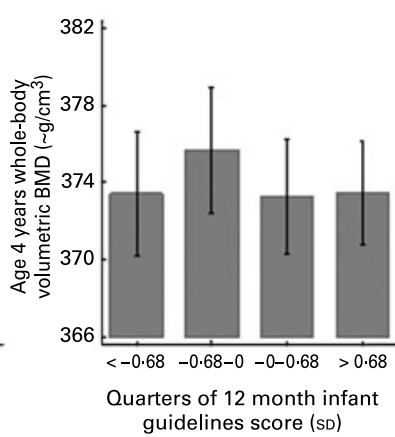

Fig. 1. (a)-(d) Duration of breast-feeding in infancy and whole-body bone mass at age 4 years among 599 children born to mothers in the Southampton Women's Survey. (e)-(h) Dietary patterns in infancy (infant guidelines score) and whole-body bone mass at age 4 years among 599 children born to mothers in the Southampton Women's Survey. Values are means, with $95 \% \mathrm{Cl}$ represented by vertical bars. BA, bone area; BMC, bone mineral content; BMD, bone mineral density.

excluded. Fourth, the use of DXA does not allow measurement of true volumetric bone density, thus making it difficult to be certain about differential determinants of skeletal size and volumetric density. Finally, it is difficult to disentangle the relative individual contributions of different dietary components in this observational study design.

Several population studies show inconsistent relationships between breast-feeding and bone mass in childhood. In a small group of low-birth-weight infants, Atkinson \& Randall-Simpson ${ }^{(20)}$ observed greater whole-body BMC relative to body size in formula-fed than breast-fed infants. Additionally, in a study of forty breast-fed and thirty-six formula-fed infants followed over the first 2 years of life, Butte et al. found reduced whole-body BMC in the breastfed $v$. formula-fed infants at 12 months $^{(1)}$. Fat mass and percentage fat mass were higher in the breast-fed infants at 3 and 6 months.

The studies above might suggest that for bone mineral accrual, formula has at least short-term benefits over breastfeeding. However, the long-term impact of milk-feeding type is also uncertain. A study of premature infants randomised to formulas of differing $\mathrm{Ca}$ concentrations or breast milk demonstrated no difference in bone mass when adjusted for body size between the different feeding regimens ${ }^{(3)}$, although they were on average shorter and lighter than children born at term. Thus those fed breast milk had normally mineralised bones for their body size. Specker et al. found that term infants fed a high-Ca formula had greater BMD than those fed breast milk at 6 months. However, when all the children were fed normal formula for the next 6 months, these differences disappeared ${ }^{(4)}$. Finally, Jones et al. ${ }^{(2)}$ studied 330 children, aged 8 years, whose feeding had been characterised in infancy. In this cohort, BMD at 8 years (measured by DXA) at femoral neck, lumbar spine and total body was higher in breast-fed infants compared with children who had been formula-fed. The association persisted after adjusting for childhood dietary and lifestyle factors and was only present for breast-feeding for more than 3 months duration in term deliveries.

There are significant nutritional differences between breast and formula milk. When compared with formula milk, breast milk has a higher content of a range of bioactive constituents ${ }^{(21)}$, but a lower content of some nutrients such as protein and vitamin D. There is some evidence to suggest that the vitamin D content of milk may be important. Two long-term follow-up studies examined the relationship between infant supplementation with vitamin $\mathrm{D}$ and childhood bone mass at age 8-9 years $^{(22,23)}$. One showed a positive association and the other demonstrated no relationship. The vitamin $\mathrm{D}$ content of breast milk depends upon the mother's circulating stores and can be increased by maternal supplementation ${ }^{(24-26)}$, but the currently available data are insufficient to definitively inform public health policy in this regard. Even earlier in the life-course, there is evidence to suggest that maternal vitamin D status in pregnancy may have a persisting influence on skeletal development in the offspring: we have previously demonstrated ${ }^{(27)}$, in an observational setting, that mothers who had low levels of circulating 25-hydroxy vitamin D in late pregnancy had children with decreased whole-body BMC at age 9 years. Additionally, we found similar results at birth in the SWS ${ }^{(28)}$. Thus maternal vitamin D status may need to be addressed in early pregnancy to maximise benefit to the offspring's skeletal development. 
Finally, in the Southampton 9 -year cohort ${ }^{(8)}$, prudent diet scores were calculated for the mothers during pregnancy, and the positive relationship of this measurement with offspring bone mass at age 9 years provides some of the very few data relating other pregnancy dietary factors to offspring bone health. Although we have previously shown that maternal prudent diet scores are highly correlated with infant guidelines pattern scores in the $\mathrm{SWS}^{(5)}$, taking account of maternal prudent diet score in our present study did not alter the findings.

\section{Conclusions}

We have demonstrated, in a prospective study, that there is no association between duration of breast-feeding or adherence to current guidelines on infant feeding with bone mineral accrual in early childhood. These findings were not modified by inclusion of maternal lifestyle and anthropometric factors, or childhood milk intake. We thus observed wide variations in current infant feeding practice, but these variations were not associated with differences in childhood bone mass at age 4 years. Future studies are needed to determine whether attention to nutritional modification to optimise bone health of the child might be most appropriately directed at mothers during pregnancy, lactation, or both.

\section{Acknowledgements}

The members of the SWS Study Group were David J. P. Barker, Catherine M. Law, Vanessa A. Cox, Elaine M. Dennison, Pat Taylor, Patricia J. Coakley and Julia Hammond.

We thank the mothers who gave us their time, I. Cameron and T. Wheeler for allowing us to include their patients, and a team of dedicated research nurses and ancillary staff for their assistance. Participants were drawn from a cohort study funded by the Medical Research Council, with additional funding from the British Heart Foundation, Dunhill Medical Trust, National Osteoporosis Society and International Osteoporosis Foundation, and Food Standards Agency contract no. N05049 for this project. We thank Mrs G. Strange for helping to prepare the manuscript.

N. C. H., Z. A. C., C. R. G. and C. C. supervised DXA assessments and 4-year follow-up. N. C. H. wrote the manuscript. S. M. R. and L. D. M. devised feeding assessments and analysis of food intake. S. R. C. provided statistical analysis. H. M. I. and K. M. G. set up and supervised the SWS cohort.

The authors declare no conflicts of interest.

\section{References}

1. Butte NF, Wong WW, Hopkinson JM, et al. (2000) Infant feeding mode affects early growth and body composition. Pediatrics 106, $1355-1366$.

2. Jones G, Riley M \& Dwyer T (2000) Breastfeeding in early life and bone mass in prepubertal children: a longitudinal study. Osteoporos Int 11, 146-152.

3. Fewtrell MS, Prentice A, Jones SC, et al. (1999) Bone mineralization and turnover in preterm infants at 8-12 years of age: the effect of early diet. J Bone Miner Res 14, 810-820.
4. Specker BL, Beck A, Kalkwarf H, et al. (1997) Randomized trial of varying mineral intake on total body bone mineral accretion during the first year of life. Pediatrics 99, E12.

5. Robinson S, Marriott L, Poole J, et al. (2007) Dietary patterns in infancy: the importance of maternal and family influences on feeding practice. Br J Nutr 98, 1029-1037.

6. Bolling K, Grant C, Hamlyn B, et al. (2007) Infant Feeding Survey 2005. London: The Information Centre.

7. Noble S \& Emmett P (2006) Differences in weaning practice, food and nutrient intake between breast- and formulafed 4-month-old infants in England. J Hum Nutr Diet 19, $303-313$.

8. Cole Z, Gale C, Javaid M, et al. (2008) Maternal dietary patterns during pregnancy and childhood bone mass: a longitudinal study. J Bone Miner Res (epublication ahead of print version 2 December 2008).

9. Inskip HM, Godfrey KM, Robinson SM, et al. (2006) Cohort profile: The Southampton Women's Survey. Int J Epidemiol 35, 42-48.

10. Robinson S, Godfrey K, Osmond C, et al. (1996) Evaluation of a food frequency questionnaire used to assess nutrient intakes in pregnant women. Eur J Clin Nutr 50, 302-308.

11. Marriott LD, Robinson SM, Poole J, et al. (2008) What do babies eat? Evaluation of a food frequency questionnaire to assess the diets of infants aged 6 months. Public Health Nutr 11, 751-756.

12. Marriott LD, Inskip HM, Borland SE, et al. (2008) What do babies eat: evaluation of a food frequency questionnaire to assess the diets of infants aged twelve months. Public Health Nutr (epublication ahead of print version 15 August 2008).

13. Joliffe IT \& Morgan BJT (1992) Principal component analysis and exploratory factor analysis. Stat Methods Med Res 1, 69-95.

14. Department of Health (2006) Birth to Five. London: Central Office of Information.

15. Robinson SM, Crozier SR, Borland SE, et al. (2004) Impact of educational attainment on the quality of young women's diets. Eur J Clin Nutr 58, 1174-1180.

16. Abrams SA, Schanler RJ, Sheng HP, et al. (1988) Bone mineral content reflects total body calcium in neonatal miniature piglets. Pediatr Res 24, 693-695.

17. Crozier SR, Inskip HM, Godfrey KM, et al. (2008) Dietary patterns in pregnant women: a comparison of food-frequency questionnaires and $4 \mathrm{~d}$ prospective diaries. $\mathrm{Br} J$ Nutr 99, $869-875$.

18. Hu FB, Rimm E, Smith-Warner SA, et al. (1999) Reproducibility and validity of dietary patterns assessed with a foodfrequency questionnaire. Am J Clin Nutr 69, 243-249.

19. Crozier SR, Robinson SM, Borland SE, et al. (2006) Dietary patterns in the Southampton Women's Survey. Eur J Clin Nutr 60, 1391-1399.

20. Atkinson SA \& Randall-Simpson J (2000) Factors influencing body composition of premature infants at term-adjusted age. Ann N Y Acad Sci 904, 393-399.

21. Hamosh M (2001) Bioactive factors in human milk. Pediatr Clin North Am 48, 69-86.

22. Zamora SA, Rizzoli R, Belli DC, et al. (1999) Vitamin D supplementation during infancy is associated with higher bone mineral mass in prepubertal girls. J Clin Endocrinol Metab 84, 4541-4544.

23. Backstrom MC, Maki R, Kuusela AL, et al. (1999) The longterm effect of early mineral, vitamin D and breast milk intake on bone mineral status in 9 to 11 -year-old children born prematurely. J Pediatr Gastroenterol Nutr 29, 575-582.

24. Hollis BW \& Wagner CL (2004) Vitamin D requirements during lactation: high-dose maternal supplementation as therapy 
to prevent hypovitaminosis $\mathrm{D}$ for both the mother and the nursing infant. Am J Clin Nutr 80, 1752S-1758S.

25. Basile LA, Taylor SN, Wagner CL, et al. (2006) The effect of high-dose vitamin D supplementation on serum vitamin D levels and milk calcium concentration in lactating women and their infants. Breastfeed Med 1, 27-35.

26. Wagner CL, Hulsey TC, Fanning D, et al. (2006) High-dose vitamin $\mathrm{D}_{3}$ supplementation in a cohort of breastfeeding mothers and their infants: a 6-month follow-up pilot study. Breastfeed Med 1, 59-70.

27. Javaid MK, Crozier SR, Harvey NC, et al. (2006) Maternal vitamin $\mathrm{D}$ status during pregnancy and childhood bone mass at age 9 years: a longitudinal study. Lancet 367, 36-43.

28. Harvey NC, Javaid MK, Poole JR, et al. (2008) Paternal skeletal size predicts intrauterine bone mineral accrual. J Clin Endocrinol Metab 93, 1676-1681. 